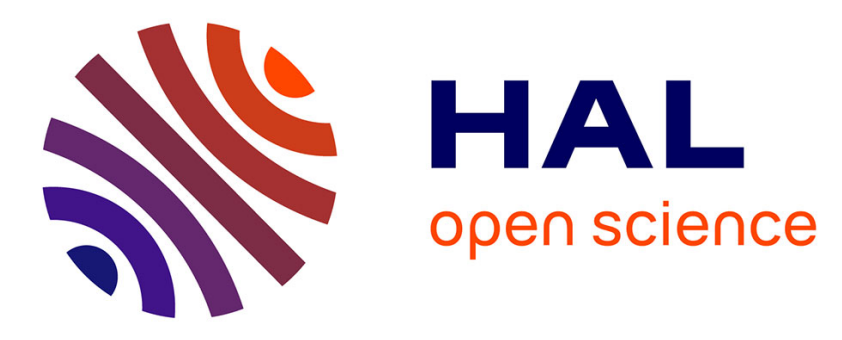

\title{
Mixed Integer Programming model for pricing in telecommunication
}

\author{
Mustapha Bouhtou, Jean-Robin Medori, Michel Minoux
}

\section{To cite this version:}

Mustapha Bouhtou, Jean-Robin Medori, Michel Minoux. Mixed Integer Programming model for pricing in telecommunication. INOC 2011 - 5th International Network Optimization Conference, Jun 2011, Hambourg, Germany. pp.626-630, 10.1007/978-3-642-21527-8_70 . hal-00703259

\section{HAL Id: hal-00703259 \\ https://hal.sorbonne-universite.fr/hal-00703259}

Submitted on 1 Jun 2012

HAL is a multi-disciplinary open access archive for the deposit and dissemination of scientific research documents, whether they are published or not. The documents may come from teaching and research institutions in France or abroad, or from public or private research centers.
L'archive ouverte pluridisciplinaire HAL, est destinée au dépôt et à la diffusion de documents scientifiques de niveau recherche, publiés ou non, émanant des établissements d'enseignement et de recherche français ou étrangers, des laboratoires publics ou privés. 


\title{
Mixed Integer Programming model for pricing in telecommunication
}

\author{
Mustapha Bouhtou and Jean-Robin Medori and Michel Minoux
}

\begin{abstract}
Yield management has been successfully applied in the context of airline companies. However, so far, application to telecommunication industry have been scarce. Using Yield management principles, this paper investigates the new problem of maximizing revenue of telecommunications operator by setting prices on voice services. This pricing is based on available resource i.e. network load. We first propose a Mixed Integer Program to model this problem. Then we study the particular case where demand and load are linear functions of price. Using numerical results, we also study the impact of a Big M constant on computational effort required to solve the problem.
\end{abstract}

\section{Introduction}

For the last three decades, yield management (YM) has attracted interest from theoretical as well as operational point of view. Generally, YM is defined as the application of information systems and pricing strategies to allocate the right capacity to the right customer at a right price and the right time in order to maximize revenue. Airlines were among the first to implement YM [5] and some other industries have chosen later to implement such a pricing system. A state of the art can be found in [4].

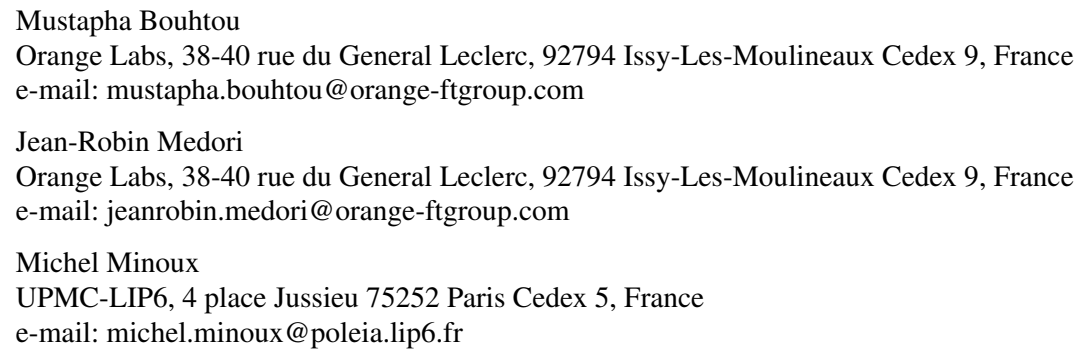


Despite this interest, so far, only a few attempts have been conducted to apply YM to the telecommunication industry. Humair [2] propose to define basis and model a framework for telecommunications. Several papers propose YM techniques to control the Internet congestion and mobile network congestion. Manaffar [3] proposes to integrate pricing with call admission control in mobile networks. Bouhtou et al. [1] studie pricing for telecommunications and propose bi-level optimization formulations to model competition between operators. Viterbo et al. [6] investigate revenue maximization in the context of a real-time pricing model for cellular networks. In this article, we deal with the new problem of pricing a voice telecommunication service provided by an operator according to available resource. In a way similar to airlines, we apply YM principles to sell voice communication on a perishable and limited resource. The main objective is revenue optimization (versus congestion control). First we formulate the problem as a Mixed Integer Program (MIP), then we study the particular case where demand and load functions are linearly dependent on price which leads to a Mixed Integer Quadratic Program (MIQP). Some preliminary numerical results are presented in section 4 .

\section{Pricing problem in voice telecommunication networks}

We consider an operator providing voice telecommunication services using a volume pricing strategy. We apply YM principles, as in airlines, and propose to set dynamic prices throughout the day, based on available resource capacity, in order to maximize daily revenue. We split a day in several time slots, with possibly different durations.

We adopt a pricing strategy based on network load. Indeed, we see in fig. 1 an example of load throughout a day at two different fixed prices. This chart shows that load is price dependent, and so the importance of choosing a pricing strategy based on network load.

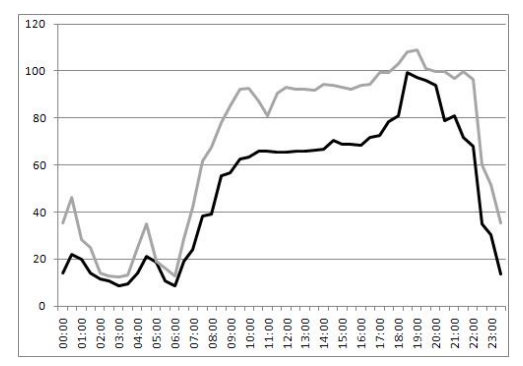

Fig. 1: Load vs time

\begin{tabular}{|c|c|c|}
\hline Load levels & Load intervals & Prices \\
\hline \hline 0 & {$\left[0 ; T h_{0}\right]$} & $r_{0}$ \\
\hline 1 & {$\left[T h_{0} ; T h_{1}\right]$} & $r_{1}$ \\
\hline$\vdots$ & $\vdots$ & $\vdots$ \\
\hline$I$ & {$\left[T h_{I-1} ; \infty[\right.$} & $r_{I}$ \\
\hline
\end{tabular}

Table 1: Price Grid

We consider several load thresholds $T h_{i}$, and the last one correspond to the network capacity. Congestion is allowed, but not in two consecutive time slots. 
In order to set a price for each time slot based on load, we assign a price to each load level. So, the daily pricing problem is reduced to finding an optimal price grid such as table 1. Price $r_{i}$ is applied at time slot $t$ if load of the previous time slot $t-1$ was in level $i$. Thus, load used to price time slot $t$ depend on price set at $t-1$. This rule makes our problem dynamic.

\section{MIP formulation}

\subsection{Notation}

The following notation is used throughout the paper :

1. Input Data :

- $T$ : Number of time slots indexed by $t$

- $I$ : Number of load thresholds indexed by $i$

- $T h_{i}$ : Load thresholds

- $p_{-1}:$ Input representing price of the last time slot of the day before

- $\rho_{t}\left(p_{t-1}\right)$ : Function representing load at $t$

- $D_{t}\left(p_{t}\right)$ : Function representing demand (number of seconds consumed) at $t$

- $R_{t}\left(p_{t}\right)$ : Function representing revenue obtained in period $t$

2. Variables :

- $p_{t} \in\left[P_{\min } ; P_{\max }\right]:$ Variable representing price assigned to time slot $t$

- $r_{i} \in\left[P_{\min } ; P_{\max }\right]$ : Variable representing price assigned to load level $i$, i.e. between $T h_{i-1}$ and $T h_{i}$

- $\beta_{i}^{t}$ : Boolean variable equals to 1 if load at $t$ is in load level $i$

- $\alpha_{i}^{t}$ : Variable equals to load at $t$ if it is in load level $i, 0$ otherwise.

\subsection{General problem formulation}

Using previous notation, we propose the general voice pricing problem $P b(M)$ based on resource capacity, and modeled as a MIP.

The objective function is taken as sum of revenues $R_{t}\left(p_{t}\right)$ on the successive periods. In constraint (2), we use $\alpha_{i}^{t}$ to model load at $t$ in load level $i$. Constraint (3) allows to set the load level in which the load $\rho_{t}$ is. Constraint (4) ensures each period to be assigned only to one load level. Constraint (5) assigns a time slot to a load level. It allows the price $r_{i}$ to be equals to price $p_{t}$ if and only if load at $t$ is in the level $i$ (i.e. $\beta_{i}^{t}=1$ ). M represents a Big M constant. Constraint (6) prevents congestion phenomenons to occur in two consecutive time slots. 


$$
\operatorname{Pb}(M)\left\{\begin{array}{rr}
\max \sum_{t=0}^{T-1} R_{t}\left(p_{t}\right) & \\
\text { subject to } & \\
\sum_{i=0}^{I} \alpha_{i}^{t}=\rho_{t}\left(p_{t-1}\right) & \forall t \\
\beta_{i}^{t} T h_{i-1} \leq \alpha_{i}^{t} \leq \beta_{i}^{t} T h_{i} & \forall i, t \\
\sum_{i=0}^{I} \beta_{i}^{t}=1 & \forall t \\
\left(\beta_{i}^{t}-1\right) M \leq p_{t}-r_{i} \leq\left(1-\beta_{i}^{t}\right) M & \forall i, t \\
\beta_{I}^{t-1}+\beta_{I}^{t} \leq 1 & \forall t \neq 0 \\
\beta_{i}^{t} \in\{0 ; 1\} & \forall i, \forall t \\
\alpha_{i}^{t} \geq 0 & \forall i, \forall t \\
r_{i} \in\left[P_{\min } ; P_{\max }\right] & \forall i \\
p_{t} \in\left[P_{\min } ; P_{\max }\right] & \forall t
\end{array}\right.
$$

\subsection{Linear demand and load model}

We focus on the special case where load and demand are linear and decreasing functions of price, and so revenue is a quadratic function of price.

$$
\begin{aligned}
\rho_{t}\left(p_{t-1}\right) & =d_{t}-c_{t} \cdot p_{t-1} \\
R_{t}\left(p_{t}\right) & =D_{t}\left(p_{t}\right) \cdot p_{t}=b_{t} \cdot p_{t}-a_{t} \cdot p_{t}^{2}
\end{aligned}
$$

where $a_{t}, b_{t}, c_{t}$ and $d_{t}$ are positive input provided by statistical data. In this case, $P b(M)$ becomes a MIQP with $T(I+1)$ binary variables, $T(I+1)+T+I+1$ continuous variables, and $4 T(I+1)+3 T-1$ linear constraints.

\section{Experimentation and numerical results}

We have built several instances with different numbers of time slots, using data measured every 30 minutes on a real network. Several tests have been performed to solve those instances using Mixed Integer quadratic programming solver from Cplex 12.1 on a workstation with 4 processors $\operatorname{Intel}(\mathrm{R}) \mathrm{Xeon}(\mathrm{R}) 1.6-\mathrm{GHz}$, and 4096-KB memory size.

In this section, we discuss the interest to consider a high number of time slots. As can be seen in fig. 2, revenue tends to increase as the number of time slots increases. The increase in optimal solution value is about $17 \%$. This confirms the importance 


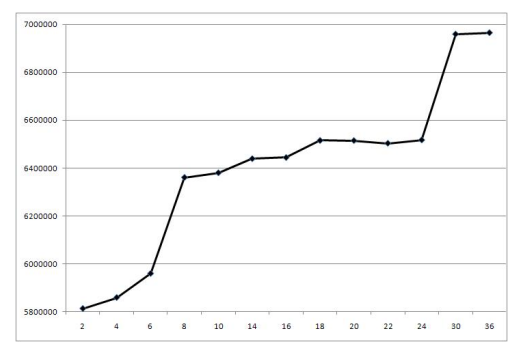

Fig. 2: Revenue vs Number of periods

to consider the problem for a sufficiently large number of time slots. However, the problem is hard to solve. Results show that for $T=8$ to $T=48$, integrity gaps become very high, between $34 \%$ and $400 \%$. Moreover, with $M=10000$, we only obtain in 4 hours, approximations with respectively for the instances with $T=42$ and $T=48$, a $1.41 \%$ and a $2.95 \%$ gaps. Thus, we have investigate ways to improve efficiency by choosing a more accurate value of the Big $M$ constant. Let $\left(p_{t}^{*}, r_{i}^{*}\right)$ be an optimal solution. we note :

$$
\begin{aligned}
& P_{\text {max }}^{*}=\max _{t}\left\{p_{t}^{*}\right\}=\max _{i}\left\{r_{i}^{*}\right\} \\
& P_{\text {min }}^{*}=\min _{t}\left\{p_{t}^{*}\right\}=\min _{i}\left\{r_{i}^{*}\right\}
\end{aligned}
$$

When $\beta_{i}^{t}=0$, equation (5) leads to $\left|p_{t}-r_{i}\right| \leq M$. As $P_{\text {max }}^{*}-P_{\text {min }}^{*}$ represents the highest difference between two prices, $M$ just have to be greater than $P_{\max }^{*}-P_{\min }^{*}$. So, the idea in the following is to derive an upper bound to $P_{\max }^{*}-P_{\min }^{*}$, i.e. upper and lower bounds respectively to $P_{\max }^{*}$ and $P_{\min }^{*}$. As a price is always positive, we consider 0 as the lower bound of $P_{\text {min }}^{*}$.

Let us define $\widehat{P_{t, i}}, \overline{P_{t}}$ and $\widetilde{P}_{t}$ such as

$$
\begin{aligned}
\rho_{t}\left(\widehat{P_{t, i}}\right) & =T h_{i} \\
\rho_{t}\left(\overline{P_{t}}\right) & =0 \\
\widetilde{P_{t}} & =\underset{p}{\arg \max }\left\{R_{t}(p)\right\}=\frac{b_{t}}{2 a_{t}}
\end{aligned}
$$

Proposition 1.

$$
P_{\max }^{*} \leq \min \left\{\max _{t}\left\{\overline{P_{t}}\right\} ; \max _{t}\left\{\widehat{P_{t, 0}} ; \widetilde{P}_{t}\right\}\right\}
$$

Proof. Because $\rho_{t}$ is positive and decreasing, $P_{\max }^{*}$ is obviously lower than each $\overline{P_{t}}$. Let us consider an optimal solution such as $P_{\max }^{*}=r_{i} \geq \max _{t}\left\{\widehat{P_{t, 0}} ; \widetilde{P}_{t}\right\}$. By contradiction, it is easy to prove that this solution is not optimal, because the solution with the same prices but $\max _{t}\left\{\widehat{P_{t, 0}} ; \widetilde{P}_{t}\right\}$ instead of $r_{i}$, is better, and still feasible (any time slot is assigned to another load level). 
We performed tests on the same instances but with $M=P_{\max }^{*}$. Results show that the choice of the $M$ value does not have any impact on integrity gap. Table 2 represents computing time and number of generated nodes according to $M$ value for some instances. Computing time and number of generated nodes are globally smaller using $M$ smaller which validate the interest of our proposition. With an accurate $M$ the instance with $T=42$ has been solved in less than 3 hours, and we obtain for the instance with $T=48$ a better approximate solution at $1.82 \%$ (vs 2.95) in about 3 hours (vs 4 hours).

\begin{tabular}{|c||c||c|c|c|c|}
\hline Number of time slots & Integrity gap & \multicolumn{2}{c|}{ Computing time in seconds } & \multicolumn{2}{c|}{ Number of generated nodes } \\
\hline & & $M=10000$ & $M=430$ & $M=10000$ & $M=430$ \\
\hline 12 & $339.65 \%$ & 0.64 & 0.33 & 509 & 408 \\
\hline 20 & $177.15 \%$ & 11.52 & 4.51 & 9000 & 7500 \\
\hline 24 & $175.84 \%$ & 26.95 & 9.98 & 19400 & 18600 \\
\hline 30 & $160.34 \%$ & 353.91 & 157.11 & 200100 & 199000 \\
\hline 42 & $396.5 \%$ & $14400[1.41 \%]$ & 9986.65 .96 & 32572500 & 11000400 \\
\hline 48 & $183.01 \%$ & $14031.41[2.95 \%]$ & $7639.57[1.82 \%]$ & $8006100[2.95 \%]$ & $7810300[1.82 \%]$ \\
\hline
\end{tabular}

Table 2: Big M influence on computing time and number of nodes

\section{Conclusion and future research work}

This paper deals with a new problem of yield management in the telecommunication industry. A MIP formulation has been proposed to model this problem of pricing voice services based on available resource. We focused our study on the particular case, where demand and load are functions linearly dependent on price resulting in a MIQP. A study on the impact of a big $M$ constant has also been carried on and led to improve the computational efficiency. In future work, we intend to extend this model, considering for example more general models for load and demand depending on several variables.

\section{References}

1. M. Bouhtou, G. Erbs and M. Minoux : Joint optimization of pricing and resource allocation in competitive telecommunications networks. Networks 50 37-49 (2007)

2. S. Humair : Yield Management for Telecommunication Networks: Defining a New Landscape. PhD thesis, Massachusetts Institue Of Technology (2001)

3. M. Manaffar, H. Bakhshi, and M. Pilevari : A new dynamic pricing scheme with call admission control to reduce network congestion. Advanced Information Networking and Applications - Workshops 347-352 (2008)

4. R. Bitran and R. Caldentey : An Overview of Pricing Models for Revenue Management. MIT Sloan Working Paper No. 4433-03 (2002)

5. B. Smith and J. Leimkuhler and R. Darrow : Yield Management at American Airlines. Interfaces (1992)

6. E. Viterbo, C.F. Chiasserini : Dynamic Pricing in Wireless Networks. (2001) 